\title{
Power Quality Comprehensive Assessment Based on Fuzzy Backward Cloud
}

\author{
Rui Zhang and Li-chuan Ma \\ School of Automation, Harbin University of Science and Technology, \\ Harbin, China \\ zr_gh@sina.com
}

\begin{abstract}
In order to improve the power quality comprehensive assessment of objectivity and accuracy, the fuzzy backward cloud generator that combines fuzzy theory and backward cloud model is proposed. Expectation of the backward cloud model is the qualitative concept that reflects the level of power quality best. The fuzzy backward cloud model using the fuzzy method to determine the numerical characteristics of cloud model for qualitative concepts of each power index, the weight of each index is determined by the combination weighting method, and then the power quality assessment is achieved. This approach is compatible with the influential factors of various levels of power quality indicators. The simulation results show the proposed method is more objective and accurate compared with the conventional method.
\end{abstract}

Keywords: power quality; fuzzy theory; combination weight; cloud model

\section{Introduction}

A large number of time-varying nonlinear devices are applied to the grid with the development of power electronics technology, and many power quality problems also appear. The power quality problem can be defined as the deviation of the current, voltage and frequency that causes the electrical equipment to fail to work correctly or to malfunction. The individual power index assessment cannot fully response the overall condition of the power quality; therefore, building a comprehensive model of power quality assessment to make a full and accurate assessment has become an urgent problem. This article will focus on voltage deviation, frequency excursion, voltage harmonic, voltage fluctuation, voltage flicker, voltage drop and three-phase unbalance seven power quality indexes to make a comprehensive assessment for a place.

The current power quality comprehensive assessment methods mainly include the method based on fuzzy theory [1], the method based on probability and statistics [2], the method based on intelligent algorithm [3]. Literature [4] proposed the assessment method based on fuzzy math, but this method is not detail, clear and comprehensive enough, this method can only get the approximate membership function in determining the maximum membership function, sometimes there will be deviation. Literature [5] proposed a method based on probability and statistics eigenvalues, using the vector mathematical method on indicators for the standardization and normalization to reduce the impact of human factors. But the shortcoming of this approach is in some special cases or power users with special needs, if still makes the variance and the mean vector normalized when different reference value selected, then the index normalized results will appear quite different. Literature [6] proposed power quality assessment method based on cloud model combining fuzziness and randomness completely, to achieve conversion between qualitative concepts and quantitative concepts. The method adopts backward cloud model of certainty, but there is no specific method to determine the 
certainty of cloud droplets.

This paper proposes a comprehensive assessment method of power quality based on fuzzy backward cloud model for the above questions, improving the method to calculate the certainty based on the membership. In order to improve the accuracy of the conclusion, it needs to calculate the single index certainty of single standard grade cloud model respectively, and using the theory of fuzzy logic to calculate the $E_{x}$ to insure the results of the cloud model parameters is more rigorous. Finally calculate similarity of the comprehensive assessment cloud and standard grade cloud based on the cloud similarity approach to get corresponding cloud model power quality grade of the tested data.

\section{Cloud Theory}

The cloud model has the uncertainty bidirectional conversion function between the qualitative concept and its quantitative concept, combining fuzziness and randomness to form mutual mapping between the qualitative and quantitative [7]. If $U$ is a quantity domain expressed with accurate numbers, and $C$ is a qualitative concept in $U$, if there is one stable random number $\mu(x) \in[0,1]$ for $x$ is a random realization of the quality concept $C$, it is the random number which has the steady tendency:

$\mu: U \rightarrow[0,1], \forall x \in U, x \rightarrow \mu(x)$

The distribution of $x$ in domain $U$ is called cloud, $x$ is called a cloud droplet [8]. The numerical characteristics of cloud model usually expressed with $\operatorname{Expectation}\left(E_{x}\right)$, $\operatorname{Entropy}\left(E_{n}\right)$ and Hyper-entropy $\left(H_{e}\right)$.Expectation $\left(E_{x}\right)$ of cloud droplets distribution in the domain space is the best representative point of the qualitative concept that reflects the gravity center of droplet swarm, is the typical sample of the concept quantization. Entropy $\left(E_{n}\right)$ reflects the range of droplets in the domain space can be accepted by the concept. Hyper entropy $\left(H_{e}\right)$ is the uncertainty measure of entropy, namely entropy of entropy, reflects the degree of condensation of droplets, the greater the entropy, the more discrete the cloud is.

\section{Fuzzy Cloud Model}

Fuzzy logic [9] is an accurate method to resolve inexact, incomplete information. Fuzzy calculation process is usually can be divided into four modules: fuzzy: the process to get fuzzy membership from the specific input according to membership function. Since the rules are expressed by the fuzzy natural language, the input is the exact numerical value, and the rules can hardly be applied without the process of fuzziness; Fuzzy rule bases: fuzzy rules provided by experts; reasoning method: the method to get fuzzy conclusion from the membership degree of correlation fuzzy sets of the fuzzy rules and input; deploring: the process to translate fuzzy conclusions into specific, precise output. The conclusion cannot be applied to the actual application without the process of the de-fuzzy. Fuzzy inference system is shown in Figure 1. 


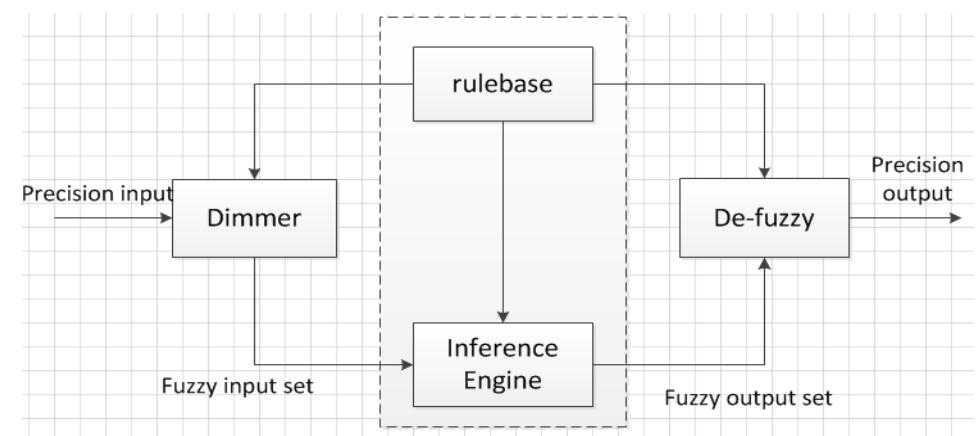

Figure 1. Fuzzy Inference System

With different reasoning method the deploring concrete steps are not the same. Common deploring method is as follows.

$$
x_{i}=\frac{\sum_{i=1}^{n} \mu_{i} \times x_{i}}{\sum_{i=1}^{n} \mu_{i}}
$$

Aiming at randomness and uncertainty of the electric energy data collection, this paper adopts fuzzy backward cloud generator to calculate cloud model feature values, constructing a comprehensive assessment cloud model of the actual data of the sampling point and obtaining the corresponding level of power quality.

\subsection{Standard Grade Cloud Model Generation Algorithm Design}

According to the assessment idea of this article, standard-grade cloud model will have a significant impact on the experiment results. Therefore, quality grade determine of each index is the key to this assessment model. The seven indicators were divided into five grades, excellent, good, medium, qualified and unqualified respectively in this paper. In order to make every standard interval has two-way binding, each index has identified a threshold value in this paper. Since these are bilateral constraints, for each standard interval $\left[R_{\min }, R_{\max }\right]$ can use the following formula torepresent:

$$
\left\{\begin{array}{c}
E_{x}=\left(R_{\min }+R_{\max }\right) / 2 \\
E_{n}=\left(R_{\max }-R_{\text {min }}\right) / 6(3) \\
H_{e}=\eta
\end{array}\right.
$$

Where $\eta$ is a constant that reflecting the randomness of the entity value, the value should not be too large. Since the larger $H_{e}$ is the greater $E_{x}$ error is and the randomness increases, it is difficult to determine the results of the assessment.

\subsection{Forward Cloud Generator}

Forward cloud generator uses the cloud numerical characteristics $\left(E_{x}, E_{n}, H_{e}\right)$ to generate $N$ droplets, completing qualitative to quantitative. The algorithm of forward cloud generator can be summed as follows:

(1) Calculate a normal random number $E_{n}^{\prime}=\operatorname{NORM}\left(E_{n}, H_{e}^{2}\right)$;

(2) Calculate a normal random number $x=\operatorname{NORM}\left(E_{x}, E_{n}^{\prime 2}\right)$;

(3) Calculate $\mu=e^{-\frac{\left(x-E_{\chi}\right)^{2}}{2\left(E_{n}^{\prime}\right)^{2}}}$;

(4) Repeat the above steps until $n$ cloud droplets.

\subsection{Fuzzy Backward Cloud Generator}

Backward cloud generator is a conversion model which can convert sample data to a quality concept with numerical characteristics $\left(E_{x}, E_{n}, H_{e}\right)$ represented, completing 
quantitative to qualitative. Using fuzzy set theory to determine different indicator when faced with different quality grade of cloud model numerical characteristics in this paper.

Assume that for $k$ energy indicators, $m$ secondary data collection for each indicator. Calculate the certainty degree of each collected data corresponding to the $q$ power quality level $\left[\mu_{1}, \mu_{2}, \mu_{3}, \ldots, \mu_{q}\right]$. Enter the expectation values of the standard grade cloud $q$ quality levels of the index to calculate the location of the expected value of the standard cloud. Using this method to calculatem expectations and calculate the mean as the desired approximation. The algorithm of fuzzy forward cloud generator can be summed as follows:

(1) Calculate the certainty of $l$ power index $x$ cloud droplets for standard level cloud $\left(E_{x}, E_{n}, H_{e}\right)$.

1) Calculate a normal random number $P_{i}=R\left(E_{n}, H_{e}^{2}\right)$;

2) Calculate $\mu_{i}=e^{-\frac{\left(x-E_{x}\right)^{2}}{2\left(P_{i}\right)^{2}}}$;

3) Repeat $n$ times and calculate the mean $\bar{\mu}_{i}$.

(2)Calculate the expectation value of $\mathrm{j}$-thsample $E_{x_{j}}=\frac{\sum_{i=1}^{q} \bar{\mu}_{i \cdot E_{x_{i}}}}{\sum_{i=1}^{q} \bar{\mu}_{i}}, j=1,2, \ldots, m$.The expectation value of the lpower index $E_{x_{l}}=\frac{1}{m} \sum_{j=1}^{m} E_{x_{j}}, l=1,2, \ldots, k$.

(3) Calculate Entropy $E_{n_{l}}=\sqrt{\frac{\pi}{2}} \times \frac{1}{N} \sum_{i=1}^{m}\left|x_{i}-E_{x_{l}}\right|, l=1,2, \ldots, k$.

(4) Calculate Hyper-entropy $H_{e_{l}}=\sqrt{\frac{1}{N-1} \sum_{i=1}^{m}\left(x_{i}-E_{x_{l}}\right)^{2}-E_{n_{l}}{ }^{2}}, l=1,2, \ldots, k$.

If $\frac{1}{N-1} \sum_{i=1}^{m}\left(x_{i}-E_{x_{l}}\right)^{2}-E_{n_{l}}{ }^{2}<0$, the $H_{e_{l}}=0$.

\subsection{Comprehensive Assessment Cloud Model}

Fuzzy backward cloud generator converts sampling cloud droplets to numerical characteristics of each attributive cloud. But due to the different importance of each power index, the weight of each attribute is also needed if make a comprehensive assessment of total power quality. The calculation steps for $k$ attribute comprehensive cloud are as follows:

(1)Calculate the weights of $k$ power indicators $\left(w_{1}, w_{2}, w_{3}, \ldots, w_{k}\right)$, and $\sum_{i=1}^{k} w_{i}=1$.

(2)Calculate numerical characteristics of comprehensive assessment cloud:

$E_{x}=\sum_{i=1}^{k} w_{i} \times E_{x_{i}}$

(4)

$E_{n}=\sqrt{\sum_{i=1}^{k} w_{i} \times E_{n_{i}}^{2}}$

(5)

$H_{e}=\sum_{i=1}^{k} w_{i} \times H_{e_{i}}$

(6)

\subsection{Similarity Calculation}

For standard cloud model $T C_{1}\left(E_{x_{1}}, E_{n_{1}}, H_{e_{1}}\right)$ andcomprehensive assessment cloud model $T C_{2}\left(E_{x_{2}}, E_{n_{2}}, H_{e_{2}}\right)$, the similarity $\delta$ of $T C_{1}$ and $T C_{2}$ is calculated as follows:

(1)Calculate a normal random number $E_{n_{i}}^{\prime}=\operatorname{NORM}\left(E_{n_{1}}, H_{e_{1}}^{2}\right)$;

(2)Calculate a normal random number $x_{i}=\operatorname{NORM}\left(E_{x_{1}}, E_{n_{i}}^{\prime 2}\right)$; 
(3)Calculate $\mu_{i}^{\prime}=e^{-\frac{\left(x_{i}-E_{x_{2}}\right)^{2}}{2\left(E_{n_{2}}\right)^{2}}}$;

(4)Repeat the above steps until $n$ times;

(5)Calculate similarity $\delta=\frac{1}{n} \sum_{i=1}^{n} \mu_{i}^{\prime}$.

\section{Combination Weighting Method}

The determination of index weight is very important to make a final accurate assessment of the power quality. In view of the features of the subjective weighting method and objective weighting method, the paper adopts the method of combination weighting method based on cooperation game theory. Its essence is a process to make the deviation between the combination weight value and the index weight value minimum.

First, calculate the subjective weight $w_{1}$ and objective weight $w_{2}$ of seven energy indicators, then the two weight vectors are combined to get the final result combining weight $w$. Any linear combination of a possible weight set can be expressed as:

$$
w^{*}=\sum_{i=1}^{2} \alpha_{i} w_{i}
$$

Where $\alpha_{i}>0, \alpha_{i}$ is weight coefficient, $w^{*}$ is a possible linear combination weight vector.

$M I N=\left\|\sum_{i=1}^{2} \alpha_{i} * w_{i}-w_{j}^{T}\right\|^{2}$

(8)

Where $j=1,2$. The model is a cross programming model, it can be converted to the optimal conditions as follows according to the differential nature of the matrix:

$\sum_{i=1}^{2} \alpha_{i} \times w_{j} \times w_{i}^{T}=w_{j} \times w_{j}^{T}$

(9)

Where $j=1,2$.The corresponding linear equations is as follows:

$\left[\begin{array}{ll}w_{1} \cdot w_{1}^{T} & w_{1} \cdot w_{2}^{T} \\ w_{2} \cdot w_{1}^{T} & w_{2} \cdot w_{2}^{T}\end{array}\right]\left[\begin{array}{l}\alpha_{1} \\ \alpha_{2}\end{array}\right]=\left[\begin{array}{l}w_{1} \\ w_{2}\end{array}\right]$

(10)

Calculate the weight coefficient, and then normalize it:

$\alpha_{i}^{*}=\frac{\alpha_{i}}{\sum_{i=1}^{2} \alpha_{i}}$

(11)

Calculate the final combination weight:

$w=\sum_{i=1}^{2} \alpha_{i}^{*} w_{i}$

\section{Simulation and Analysis}

(1) In order to verify the validity of the power quality assessment method based on fuzzy backward cloud proposed in this paper, using the sample data in literature [10]. We treat $w_{1}$ as the subjective weight value, treat $w_{2}$ as objective weight value and calculate them. Optimize the combination weight according to the equation (7) (12). The combination weight $w$, subjective weight $w_{1}$, objective weight $w_{2}$ and the combination weight in literature [10] are shown in Table 1. 
Table 1.Comparison of Calculation Index Weights of Various Methods

\begin{tabular}{c|c|c|c|c|c|c|c}
\hline Method & $\begin{array}{c}\text { Voltage } \\
\text { deviation }\end{array}$ & $\begin{array}{c}\text { Frequency } \\
\text { excursion }\end{array}$ & $\begin{array}{c}\text { Voltage } \\
\text { harmonic }\end{array}$ & $\begin{array}{c}\text { Voltage } \\
\text { fluctuation }\end{array}$ & $\begin{array}{c}\text { Voltage } \\
\text { flicker }\end{array}$ & $\begin{array}{c}\text { Voltage } \\
\text { drop }\end{array}$ & $\begin{array}{c}\text { Three-phase } \\
\text { unbalance }\end{array}$ \\
\hline Subjective method & 0.15281 & 0.16067 & 0.1427 & 0.14831 & 0.14157 & 0.12135 & 0.13258 \\
\hline $\begin{array}{c}\text { Objective } \\
\text { method }\end{array}$ & 0.2278 & 0.30303 & 0.08464 & 0.16614 & 0.1139 & 0.06486 & 0.0397 \\
\hline $\begin{array}{c}\text { Method of literature } \\
{[10]}\end{array}$ & 0.20833 & 0.3141 & 0.09722 & 0.16239 & 0.10791 & 0.06197 & 0.04807 \\
\hline $\begin{array}{c}\text { Paper combination } \\
\text { method }\end{array}$ & 0.20649 & 0.26585 & 0.10113 & 0.16107 & 0.12176 & 0.080856 & 0.066093 \\
\hline
\end{tabular}

From Table 1, the order of the weight results based on the combination method of cooperation game theory are consistent with the results in literature [10], it demonstrates the effectiveness of this method. Due to the method of cooperation game theory based on the idea of minimizing the sum of weighted deviation, so it has the characteristics of both subjective and objective weighting methods.

(2) Dividing the quality grade and constructing the standard grade cloud model. The grade of each power quality indicator is shown in Table 2.

Table 2.Power Quality Assessment Index Grade Emarcation Table

\begin{tabular}{l|c|c|c|c|c}
\hline Assessment index & Excellent & Good & Medium & Qualified & Unqualified \\
\hline Voltage deviation\% & $(0,1.2)$ & $(1.2,3)$ & $(3,4.5)$ & $(4.5,7)$ & $(7,10)$ \\
\hline Frequency excursion Hz & $(0,0.05)$ & $(0.05,0.1)$ & $(0.1,0.15)$ & $(0.15,0.2)$ & $(0.2,0.4)$ \\
\hline Voltage harmonic\% & $(0,1)$ & $(1,2)$ & $(2,3)$ & $(3,5)$ & $(5,8)$ \\
\hline Voltage fluctuation \% & $(0,0.5)$ & $(0.5,1)$ & $(1,1.5)$ & $(1.5,2)$ & $(2,4)$ \\
\hline Voltage flicker \% & $(0,0.2)$ & $(0.2,0.5)$ & $(0.5,0.8)$ & $(0.8,1)$ & $(1,2)$ \\
\hline Voltage drop & $(1,0.9)$ & $(0.9,0.8)$ & $(0.8,0.5)$ & $(0.5,0.1)$ & $(0.1,0)$ \\
\hline Three-phase unbalance\% & $(0,0.5)$ & $(0.5,1)$ & $(1,1.5)$ & $(1.5,2)$ & $(2,4)$ \\
\hline
\end{tabular}

Normalize the data in Table 2 and calculate numerical characteristics of the standard cloud by formula (3), let $\eta=0.04$. Obtain weighted standard cloud according to the combination weight results of table 1 . As shown in Table 3.

Table 3. Power Quality Assessment Index Grade Cloud Model

\begin{tabular}{c|c|c|c|c|c}
\hline Assessment Index & Excellent & Good & Medium & Qualified & Unqualified \\
\hline \multirow{2}{*}{ Voltage deviation\% } & $(0.06$, & $(0.21$, & $(0.375$, & $(0.575$, & $(0.85$, \\
& $0.02)$ & $0.03)$ & $0.025)$ & $0.04167)$ & $0.05)$ \\
\hline \multirow{2}{*}{ Frequency excursion Hz } & $(0.0625$, & $(0.1875$, & $(0.3125$, & $(0.4375$, & $(0.75$, \\
& $0.0208)$ & $0.02083)$ & $0.02083)$ & $0.02083)$ & $0.08333)$ \\
\hline \multirow{2}{*}{ Voltage harmonic\% } & $(0.0625$, & $(0.1875$, & $(0.3125$, & $(0.5$, & $(0.8125$, \\
& $0.02083)$ & $0.02083)$ & $0.02083)$ & $0.04167)$ & $0.0625)$ \\
\hline \multirow{2}{*}{ Voltage fluctuation \% } & $(0.04$, & $(0.165$, & $(0.3125$, & $(0.4375$, & $(0.75$, \\
& $0.01333)$ & $0.02833)$ & $0.02083)$ & $0.02083)$ & $0.08333)$ \\
\hline \multirow{2}{*}{ Voltage flicker \% } & $(0.0025$, & $(0.13$, & $(0.325$, & $(0.45$, & $(0.75$, \\
& $0.001667)$ & $0.04)$ & $0.025)$ & $0.01667)$ & $0.08333)$ \\
\hline \multirow{2}{*}{ Voltage drop } & $(0.95$, & $(0.85$, & $(0.65$, & $(0.3$, & $(0.05$, \\
& $0.01667)$ & $0.01667)$ & $0.05)$ & $0.06667)$ & $0.01667)$ \\
\hline \multirow{2}{*}{ Three-phase unbalance\% } & $(0.04$, & $(0.85$, & $(0.65$, & $(0.3$, & $(0.05$, \\
& $0.01333)$ & $0.01667)$ & $0.05)$ & $0.06667)$ & $0.01667)$ \\
\hline Weighted comprehensive & $(0.12133$, & $(0.2336$, & $(0.35421$, & $(0.46261$, & $(0.72053$, \\
standard cloud & $0.017423)$ & $0.026151)$ & $0.025762)$ & $0.033762)$ & $0.071972)$ \\
\hline
\end{tabular}


(3)Gathering information and preprocessing. For verification and comparison with the proposed method, adopting data from literature [10].Collect and normalization 8 sets of data for each power index. As shown in Table 4.

Table 4. Normalized Power Quality Measurement Data

\begin{tabular}{l|c|c|c|c|c|c|c|c}
\hline Voltage deviation & 0.4797 & 0.168 & 0.435 & 0.633 & 0.322 & 0.469 & 0.474 & 0.62 \\
\hline Frequency excursion & 0.305 & 0.155 & 0.45 & 0.4425 & 0.255 & 0.345 & 0.36 & 0.465 \\
\hline Voltage harmonic & 0.34 & 0.16 & 0.5834 & 0.42 & 0.3213 & 0.4136 & 0.3488 & 0.5075 \\
\hline Voltage fluctuation & 0.3325 & 0.1325 & 0.4875 & 0.3425 & 0.22 & 0.4175 & 0.365 & 0.3925 \\
\hline Voltage flicker & 0.27 & 0.165 & 0.465 & 0.42 & 0.16 & 0.33 & 0.285 & 0.38 \\
\hline Voltage drop & 0.524 & 0.759 & 0.356 & 0.173 & 0.623 & 0.313 & 0.176 & 0.192 \\
\hline Three-phase unbalance & 0.2075 & 0.09 & 0.3375 & 0.435 & 0.2075 & 0.285 & 0.2725 & 0.335 \\
\hline
\end{tabular}

Calculate numerical characteristics of assessment cloud by the fuzzy backward cloud generator, and generate comprehensive assessment cloud by formula (4) - (6). Results are shown in Table 5.

Table 5. Numerical Characteristic Value of Assessment Cloud

\begin{tabular}{c|c|c|c|c|c|c|c|c}
\hline & $\begin{array}{c}\text { Voltage } \\
\text { deviation }\end{array}$ & $\begin{array}{c}\text { Frequency } \\
\text { excursion }\end{array}$ & $\begin{array}{c}\text { Voltage } \\
\text { harmonic }\end{array}$ & $\begin{array}{c}\text { Voltage } \\
\text { fluctuation }\end{array}$ & $\begin{array}{c}\text { Voltage } \\
\text { flicker }\end{array}$ & $\begin{array}{c}\text { Voltage } \\
\text { drop }\end{array}$ & $\begin{array}{c}\text { Three-phase } \\
\text { unbalance }\end{array}$ & $\begin{array}{c}\text { Comprehensive } \\
\text { assessment } \\
\text { cloud }\end{array}$ \\
\hline $\boldsymbol{E}_{\boldsymbol{x}}$ & 0.47992 & 0.34136 & 0.39115 & 0.32766 & 0.3071 & 0.42784 & 0.27231 & 0.37105 \\
\hline $\boldsymbol{E}_{\boldsymbol{n}}$ & 0.12924 & 0.10415 & 0.11819 & 0.10565 & 0.11201 & 0.24309 & 0.096408 & 0.12817 \\
\hline $\boldsymbol{H}_{\boldsymbol{e}}$ & 0.08497 & 0.02741 & 0.04895 & 0,03965 & 0.02051 & 0 & 0.04033 & 0.04124 \\
\hline
\end{tabular}
2 .

(4)Comprehensive assessment cloud and standard grade clouds are shown in Figure

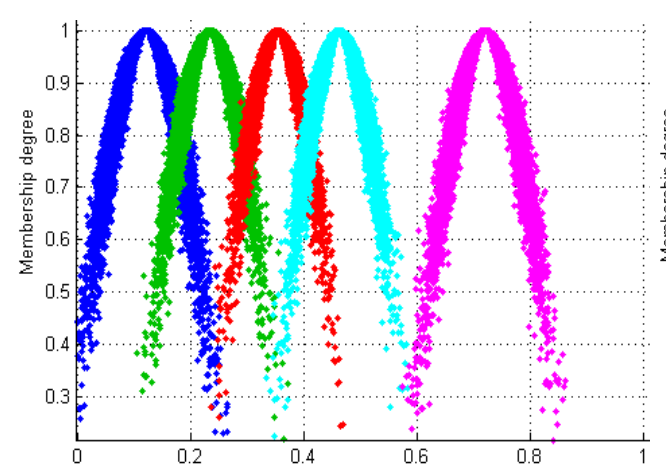

Standard grade clouds

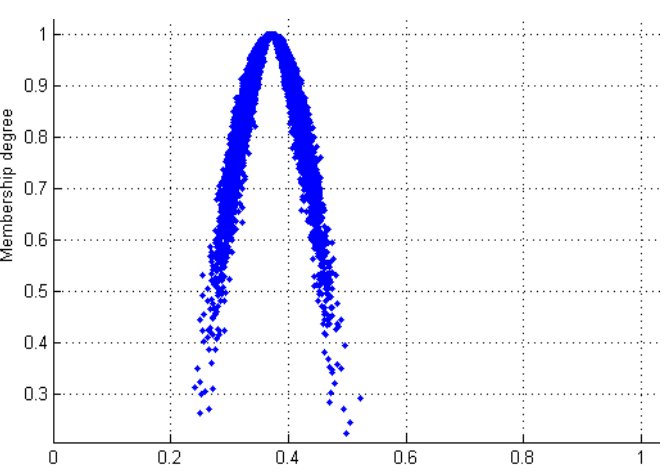

Comprehensive assessment cloud

\section{Figure 2. Standard Grade Clouds and Comprehensive Assessment Cloud}

From Figure 2 it can initially determine the level of power quality is closest to the middle, near qualified.

(5)Calculate the similarity value of the comprehensive assessment cloud model of the five standard grade clouds by similarity algorithm to get the quantitative results. As shown in Table 6.

Table 6. Comprehensive Assessment Cloud of Similarity of Standard Grade Clouds

\begin{tabular}{c|c|c|c|c|c}
\hline & Excellent & Good & Medium & Qualified & Unqualified \\
\hline Similarity & 0.03747 & 0.14066 & 0.24785 & 0.19372 & 0.00613 \\
\hline
\end{tabular}


From the results shown in Table 6, droplets distribution cloud and the medium power quality grade cloud of the highest similarity, the cloud droplets in the medium quality and below grades accounted for $71.54 \%$ of total cloud droplets. It can be judged that the power quality grade of the area belongs to medium deviation.

(6) Comparison with the traditional backward cloud generator. Cloud expectation is a decisive factor for the determination of power level in power quality comprehensive assessment based on cloud model. Therefore, to ensure the accuracy of expectation is very important. In order to verify the accuracy of the fuzzy backward cloud generator used in this paper, select the cloud model $(5,30,1)$, to generate a plurality of cloud droplets with forward cloud generator first, then using traditional backward cloud generator and fuzzy backward cloud generator to calculate expectation respectively. The simulation results are shown in Table 7.

Table 7. Two Kinds of Backward Cloud Generator $\mathbf{E}_{\mathbf{x}}$ Comparison

\begin{tabular}{l|l|l|l|l}
\hline Backward cloud algorithm & 1000 & 2000 & 4000 & 8000 \\
\hline Traditional algorithm & 29.7981 & 30.0329 & 29.9881 & 30.0017 \\
\hline Proposed algorithm & 29.8368 & 30.0023 & 30.0009 & 29,9999 \\
\hline
\end{tabular}

From the results shown in Table 7, it can see that with the increase of the number of cloud droplets, the expectation value is more and more close to the actual result. The expectation result is more accurate with fuzzy backward cloud generator than traditional backward cloud generator in the same number cloud droplets. It proved that fuzzy backward cloud algorithm is effective; the experimental results obtained are reliable.

\section{Conclusions}

In this paper, fuzzy theory is used to determine cloud model eigenvalues, forming fuzzy backward cloud generator. Using the fuzzy reasoning to structure characteristics of the cloud mode according to the test samples for each grade of memberships. Using a combination weighting method to determine the weights to obtain a comprehensive assessment of power quality cloud model. Simulation results show that the proposed assessment method not only improve the accuracy of the eigenvalues of the cloud model, but more objectively reflect the power quality of the measured area, to achieve the correct assessment of power quality.

\section{Acknowledgements}

This work was supported by Harbin Technology Innovation Talents Special Foundation of China (2014RFQXJ024).

\section{References}

[1] C. Cong, C. Hong-kun, "Fuzzy Theory in Power Quality Evaluation. Proceedings of the 7th Power Quality Symposium”, Sichuan, China, (2014) August, pp. 356-363.

[2] L. Dan, P. Ke, "Assessment Based on Power Quality Probability Theory and Fuzzy Mathematics", Theory of Urban Construction, vol. 15, (2011).

[3] Z. Lin, L-h Zhang Feng, "Genetic Projection Pursuit Interpolation Model in the Application of Power Quality Comprehensive Assessment", Power Grid Technology, vol. 7, no. 31, (2007).

[4] W. Jing, Y. Hong-li, S. Yu, "Fuzzy Attribute Analysis of Power Quality", Shaanxi Electric Power, vol. 12, (2010).

[5] G. Wen, W. Hui-xiang, Y. Cui-lan, "Based on the Probability and The Vector Algebra of Power Quality Comprehensive Assessment", Journal of Shanxi Electric Power, vol. 6, (2009).

[6] W. Hui, Q. Shu, L. Shao-ying, "Reverse Cloud Curve Fitting Improved Algorithm", CAAI Transactions 
on Intelligent Systems, vol. 5, no. 9 (2014).

[7] Li De-yi, "Membership Clouds and Cloud Generators". The Research and Development of Computers, vol. 6, no. 32, (1995).

[8] Y. Qiong, L. Shao-wen, Z. You-hua, "Overview of Cloud Models and Applications", Computer Engineering and Design, vol. 32, no. 12, (2011).

[9] P. Dao-wu, "Basic Theory of Fuzzy Reasoning", University Application Mathematics Journal, vol. 3, no. 27, (2012).

[10] L.Guo-dong, L. Geng-yin, Y. Xiao-dong, “The Power Quality Comprehensive AssessmentModel Based on Radar Map Method", Automation of Electric Power Systems, 14, (2010). 
International Journal of Grid and Distributed Computing Vol. 9, No. 2 (2016) 